Paranà (UFPR), Curitiba, Brazil, and the Fondazione IRCCS Ca' Granda, Milan, Italy.

Methods The objects of the joint project include:

- a cohort mortality study among asbestos-cement workers in the Curitiba Metropolitan Region;

- the implementation of a registry of malignant mesothelioma (MM) in Curitiba based on the Italian model.

MM cases are already collected (1998-2012) in the Curitiba Population-Based Cancer Registry (RCBP); but information on asbestos exposure is unavailable and diagnostic accuracy of MM has never been evaluated. For this reason, we translated the Italian standardised questionnaire on asbestos exposure and are implementing the database for $\mathrm{MM}$ cases in Portuguese. Clinical documentation of adult subjects with ICD-10 codes C45 (mesothelioma), C38 (mediastinal and pleural cancers), and C48 (peritoneal cancers) in the RCBP will be examined to evaluate diagnostic accuracy.

Results The questionnaire has been preliminarily tested on 40 workers in Curitiba. The software has been translated and adapted. Review of clinical records of 269 subjects is in progress; we identified about $20 \%$ of death certificate only cases. For the cohort study, we obtained lists of workers employed since the beginning of production in three asbestos-cement factories. We identified about 4000 workers, 2300 with valid date of birth.

Conclusion For the cohort study, we are exploring the existence of additional sources to complete missing information and contacting relevant institutions to perform the mortality follow-up. Within 2018 we foresee to have the questionnaire ready for use, the software implemented, and the clinical documentation review completed. This international collaboration is an important step towards a better assessment of the impact of chrysotile use in Brazil.

\section{NIGHT SHIFT WORK, BODY WEIGHT GAIN AND OBESITY OCCURRENCE: PRELIMINARY RESULTS WITH 3 YEARS OF FOLLOW-UP}

${ }^{1}$ Lap Ah Tse*, ${ }^{2}$ Zhimin Li, ${ }^{2}$ LiuZhuo Zhang, 'Miaomiao Sun, ${ }^{1}$ Feng Wang. 'Division of Occupational and Environmental Health, JC School of Public Health and Primary Care, The Chinese University of Hong Kong, Hong Kong SAR, China; ${ }^{2}$ Shenzhen Prevention and Treatment Centre for Occupational Diseases, Shenzhen, Chin

\subsection{6/oemed-2018-ICOHabstracts.397}

Introduction Previous studies in Asia developed areas showed inconsistent findings on the association between nightshift work and obesity occurrence, and there has been no study in Chinese. This study aims to examine the association between nightshift work, body weight gain and obesity occurrence in a Chinese night shift cohort after 3 years of follow-up.

Methods We recruited 5256 male workers from 6 companies in China in 2013. We used standardised questionnaire to collect participants' information on occupational history of shift work. Night shift work was defined as ever worked in a working schedule during 00-5 am at least once per month for no less than one year. Anthropometric parameters were measured using standard medical protocols. All participants were followed up till the end of 2017. Multiple logistic regression analysis was conducted to evaluate the association between night shift work and overweight (BMI $\geq 25$ ), obesity (BMI $\geq 30$ ) and BMI gain status.
Results We only included 492 male workers in this report, as the data of other participants have not been input whilst the overall results from entire cohort will be presented in $\mathrm{ICOH}$ 2018. The mean age of night shift work and daytime workers is comparable ( 30 vs 29 years). Compared with the daytime workers, slightly more night shift workers were current smokers $(25.1 \%$ vs $23.7 \%)$ and alcohol drinkers $(28.1 \%$ vs $22.2 \%)$, but fewer had vigorous physical activity (29.9\% vs $59.4 \%$ ). More nightshift workers than daytime workers slept less than 8 hours per night $(60.5 \%$ vs $34.8 \%)$ but the proportion of working longer than 55 hours per week was substantially higher $(6.6 \%$ vs $3.7 \%)$. After three years of follow-up, night shift workers showed more BMI gain than the daytime workers $(1.45 \pm 1.10$ vs $1.32 \pm 1.09)$. More night shift workers with large body mass index at baseline tended to retaining in the same category of overweight (BMI $\geq 24 \mathrm{~kg} / \mathrm{m}^{2}$ ) status with odds ratios of 1.49 (95\% CI: 0.95 to 2.33 ), and stayed in the same category of abdominal obesity with OR of $1.34(95 \%$ CI: 0.86 to 2.11). More night shift workers developed abdominal obesity from normal body size during 3 year period of follow-up and the risk of abdominal obesity was 1.43 (95\% CI: 0.75 to 2.73 ), but there was no statistical significance.

Conclusions This study provided preliminary evidence to on a possible link between nightshift work and obesity occurrence or body weight gain in Chinese male workers; however, these findings would be verified in a larger dataset of all 5256 workers.

Funding This work was supported by National Natural Science Foundation of China (Project number 81273172 and 81372964).

\section{THE PREVALENCE AND RISK OF MUSCULOSKELETAL DISORDERS IN DENTAL TECHNICIANS IN SOUTH AFRICA}

Joy Adetiba, Penny Orton*, Babusisiwe Kumalo. Durban University of Technology, Durban, South Africa

\subsection{6/oemed-2018-ICOHabstracts.398}

Introduction Musculoskeletal disorders (MSDs) affect the health, productivity and careers of the working population. Disorders of the musculoskeletal system are some of the most common occupational diseases and injuries. Dental technicians are at risk of MSDs as much as other members of the dental professionals as a result of their daily activities which are labour intensive and involve manual work. The risk factors for MSDs among dental professionals are multifactorial.

Methods Quantitative research using a cross sectional correlational survey design was adopted. A purposive sample of 79 technicians was used with 72 valid questionnaires available for analysis. A modified Nordic musculoskeletal questionnaire was used to collect data. Statistical analysis, using SPSS 23 included frequencies, chi square test of independence, independent samples t-test and Pearson's correlation.

Result The 12 month prevalence of MSDs for dental technicians in South Africa was high (90\%). For the different body regions, the prevalence ranged from 59.7\%-68.1\% and the body parts commonly affected are the neck (68.1\%), shoulders (59.7\%), wrists/hands (68.1\%), upper back (68.1\%) and lower back $(68.1 \%)$. Factors that were identified to be strongly associated with MSDs among dental technicians in South Africa are age, years of practice, standing and vibration. 
Discussion Studies show that there is a high prevalence of MSDs among dental professionals all over the world, which is related to their work environment, individual characteristics of the worker and the ergonomics of dental practice. There are many opportunities to assist dental professionals, particularly the dental technicians, to maintain optimal health during the course of their career. This could be done through improved occupational health practice, education on risk factors for MSDs, adjustment of the work environment and wellness maintenance. These measures will improve the quality of life, and ensure that older technicians do not leave the profession due to occupational injuries.

\section{ANXIETY AMONG HEALTH CARE WORKERS OF A TEACHING HOSPITAL}

R Pougnet, G Verdier, P Saliou, F Dantec, B Eniaffe, Q Durand-Moreau, B Loddé, JD Dewitte. Teaching Hospital Of Brest, Brest, France

\subsection{6/oemed-2018-ICOHabstracts.399}

An investigation in psychological suffering in all hospital workers of a teaching hospital has been conducted during one month.

Population and methods Our population was composed of 5792 non medical professionals of a teaching universitary hospital. 1738 workers were randomly selected, and answered an online auto-questionnaire, consisting of 4 parts: socio-professional data; JCQ questionnaire (Karasek); HAD questionnaire and working experience scale. Data were collected on Sphinx Online R software and exploited on Excel, using Chi2 statistical test with $\mathrm{p}<0.05$.

Results In this study, 822 questionnaires were exploitable, representing $47.3 \%$ of the population, and including $81.3 \%$ of women. The mean age was 41.8 years old, and workers were $84.3 \%$ permanent staff. Job strained staff represented $51.7 \%$ of the population, whereas $33.7 \%$ of the staff were in active. The overall isostrain was $31.6 \%$. More permanent workers, nurse's aide and hospital keener were job strained $(\mathrm{p}<0.05)$. Depression prevalence was of $8 \%$ : men, technical agents and people between 42 and 50 years old had a higher risk of depression, respectively 13\%, 15.3\% and $13.4 \% \quad(\mathrm{p}<0.05)$. Anxiety prevalence was of 21.6\%: agents before 42 and 50 years old, permanent workers, nurse's aide, had an increased risk of presenting anxious disorder, respectively 28.2\%, 22.9\% and $28 / 3 \%(\mathrm{p}<0.05)$.

Conclusion Anxiety and depression prevalences were important in this study, in a hospital environment, and were higher than in general population. High level of psychological demand partly explained those prevalences.

\section{WORK-RELATED VIOLENCE AMONG YOUNG NIGHT WORKERS IN SOUTH KOREA}

\footnotetext{
${ }^{1}$ Jia Ryu*, ${ }^{2}$ Hyunjoo Kim, ${ }^{3}$ Jaemin Kim, ${ }^{1}$ Yeogyeong Yoon, ${ }^{1}$ Kyunghee Jung-Choi. ${ }^{1}$ Department of Occupational and Environmental Medicine, College of Medicine, Ewha Womans University, South Korea; ${ }^{2}$ Department of Occupational and Environmental Medicine, Ewha Womans University Mokdong Hospital, South Korea; ${ }^{3}$ Seoul Labour Centre, South Korea
}

10.1136/oemed-2018-ICOHabstracts.400
Introduction In the 24 hour society, night workers encounter more chance of violence. This study aimed to investigate the prevalence of work-related violence and the mental health problems related to the work-related violence of young adults who worked as night workers in South Korea.

Methods The survey was conducted among 507 night workers aged 19-29 who worked in convenience stores, coffee shops, and fast-food restaurants in Seoul, South Korea. The data were obtained by well-trained interviewers with a structured questionnaire. Work-related violence was defined as a physical attack, verbal abuse, sexual harassment, and bullying that occurred in work-related situations or circumstances. The risks of post-traumatic stress disorder, insomnia, and depression were measured using the impact event scale-revised scores, insomnia severity index, and PHQ-9, respectively.

Results Among total 507 night workers included in the study, 159 (31.4\%) had experienced at least one violent incident during their work hours. Among workers who had experienced violence in the workplace, verbal abuse was the most common type. The prevalence of work-related violence increased with longer night working time and longer tenure. Regardless of the type of violence, most of the perpetrators were found to be customers. We observed that $27(17.0 \%)$ of the workers who had experienced violence were at high risk of post-traumatic stress disorder (PTSD). Furthermore, the night workers who had experienced sexual harassment and bullying were shown to have a $41 \%$ and $67 \%$ higher risk of PTSD, respectively. In addition, we found that night workers who had experienced work-related violence were subject to 3.0 and 3.2 times increased risk of insomnia (95\% CI: 1.98 to 4.62 ) and depression (95\% CI: 1.91 to 5.38), respectively.

Conclusion Work-related violence which night workers experienced could be a risk factor for mental illness. Protective intervention against violence toward night workers is urgently needed.

\section{THE INFLUENCE OF WORKING HOURS ON DEPRESSIVE SYMPTOMS IN SOUTH KOREA}

${ }^{1}$ Yeogyeong Yoon*, '1ia Ryu, ${ }^{2}$ Hyunjoo Kim, ${ }^{1}$ Kyunghee Jung-Choi. 'Department of Occupational and Environmental Medicine, College of Medicine, Ewha Womans University, South Korea; ${ }^{2}$ Department of Occupational and Environmental Medicine, Ewha Womans University Mokdong Hospital, South Korea

\subsection{6/oemed-2018-ICOHabstracts.401}

Introduction South Korea is one of the countries with the longest working hours in the OECD countries. Long working hours can be a risk factor of mental illness as well as cardiovascular disease. The aim of this study was to evaluate the effect of long working hours on depressive symptoms among employees in South Korea.

Methods This study used data from the Korea Working Conditions Survey in 2014. Study subjects included 22910 employees aged 19 years or older who work more than 35 hours per week. Working hours were categorised into 35$40,41-52,53-68$, and more than 68 hours per week. Depressive symptoms were assessed using the WHO's Well-Being Index with cut-off score of 50. We calculated prevalence ratios of depressive symptoms according to working hours using log-binomial regression.

Results Only 38.6\% of Korean employees worked 35-40 hours per week. The proportion of employees who worked 41-52, 53-68, and more than 68 hours were 32.7, 15.4, and 4.7\%, 